\title{
Recommandations pour la mise en place d'études multicentriques avec IRM
}

Recommendations for the implementation of multicenter studies with MRI

Titre court :

Recommandations Études Multicentriques IRM

Nom des auteurs et affiliations

Gabriela HOSSU ${ }^{1,2 *}$, Irène TROPRÈS ${ }^{3 *}$, Emmanuelle LE BARS ${ }^{4}$, Jean-Luc ANTON ${ }^{5}$, Marie CHUPIN $^{6,7,8,9,10 *}$, Elise BANNIER ${ }^{11,12 *}$ et le réseau REMI

- G. H et I. T. Co-premiers auteurs

- M. C et E. B Co-derniers auteurs

\section{Auteur correspondant :}

Elise BANNIER

elise.bannier@,irisa.fr

Affiliations

${ }^{1}$ Université de Lorraine, Inserm, IADI, F-54000 Nancy, France

${ }^{2}$ CHRU-Nancy, Inserm, Université de Lorraine, CIC, Innovation Technologique, F-54000 Nancy, France

${ }^{3}$ Univ. Grenoble Alpes, Inserm, CHU Grenoble Alpes, CNRS, IRMaGe, 38000 Grenoble, France

${ }^{4}$ Département de Neuroradiologie, Centre hospitalier Universitaire Montpellier, I2FH, Institut d'Imagerie Fonctionnelle Humaine, Hôpital Gui de Chauliac, 80 Avenue Augustin Fliche, 34295 Cedex 5, Montpellier, France. 
${ }^{5}$ Aix-Marseille Université, CNRS, Centre IRM-INT@CERIMED, INT UMR 7289, Marseille, France

${ }^{6}$ Paris Brain Institute - Institut du Cerveau (ICM), F-75013, Paris, France

${ }^{7}$ Inserm, U 1127, F-75013, Paris, France

${ }^{8}$ CNRS, UMR 7225, F-75013, Paris, France

${ }^{9}$ Sorbonne Université, F-75013, Paris, France

${ }^{10} \mathrm{CATI}$, Paris, France

${ }^{11}$ Univ Rennes, CNRS, Inria, Inserm, IRISA UMR 6074, Empenn - ERL U 1228, F-35000

Rennes, France

${ }^{12}$ CHU Rennes, Service de Radiologie, Plateforme Neurinfo, 2 rue Henri Le Guilloux, 35033

Rennes Cedex, France 


\section{Résumé en Français}

L'IRM prend une place de plus en plus grande dans les études de recherche clinique, que ce soit au niveau des critères d'inclusion ou des critères d'évaluation. Des marqueurs basés sur l'exploitation des acquisitions IRM sont désormais considérés régulièrement comme critères principaux d'évaluation. Dans le cadre d'études multicentriques en particulier, la gestion des acquisitions IRM doit considérer l'hétérogénéité des systèmes IRM en termes de constructeurs, de champs magnétiques, d'antennes de réception et de versions logicielles. Cela s'explique par le nombre de paramètres importants qu'il est possible de spécifier pour obtenir une acquisition IRM, la variabilité entre les solutions proposées par les constructeurs et les innovations techniques régulières.

L’objectif du présent article est de détailler les spécificités à prendre en compte et les personnes à impliquer pour réaliser une étude en IRM quel que soit l'organe concerné par l'imagerie. En s'appuyant sur l'expérience et l'expertise des membres du Réseau d'Entraide Multicentrique en IRM (REMI), nous proposons à la communauté francophone des recommandations détaillées, afin de mieux prendre en compte les spécificités de l'IRM à toutes les étapes de l'étude et de participer à l'amélioration de la qualité de la recherche en IRM. Ces recommandations prennent également en compte les spécificités des plateformes de recherche en IRM et des services d'imagerie clinique, ainsi que les contraintes réglementaires.

Mots clés : Recherche clinique, IRM, Multicentrique 


\section{Résumé en Anglais}

MRI is becoming increasingly important in clinical research studies, both in terms of inclusion criteria and endpoints. Markers based on MRI acquisitions are now regularly considered as primary endpoints. Especially in multicenter studies, the management of MRI acquisitions has to take into account the heterogeneity of MRI systems in terms of manufacturers, magnetic fields, coils and software versions. This is due to the number of important parameters that can be specified and optimised to obtain MR images, the variability between the solutions proposed by the manufacturers and the regular technical innovations.

The aim of this article is to detail the steps and the people to involve in order to carry out an MRI study. Based on the experience and expertise of the members of the Réseau d'Entraide Multicentrique en IRM (REMI), we propose detailed recommendations to the French-speaking community, in order to better take into account the specificities of MRI at all stages of the study and to participate in improving the quality of MRI research. These recommendations also take into account the specificities of MRI research platforms and clinical imaging services, as well as regulatory constraints.

Keywords: Clinical research, MRI, Multicenter 


\section{$\underline{\text { Points essentiels }}$}

Des marqueurs basés sur l'exploitation des acquisitions IRM sont désormais utilisés comme critères principaux d'évaluation dans les études de recherche clinique.

Dans le cadre d'études multicentriques, la gestion des acquisitions IRM doit considérer l'hétérogénéité des systèmes IRM en termes de constructeurs, de champs magnétiques, d'antennes et de versions logicielles.

Tous les acteurs doivent être associés dès le montage du projet.

Outre la définition du protocole IRM et sa faisabilité en multicentrique, l'identification des ressources matérielles et humaines nécessaires ainsi que le coût associé, la collecte des données, le contrôle de leur conformité, le traitement et l'analyse des données sont à considérer. 


\section{Introduction}

L'IRM prend une place de plus en plus grande dans les études de recherche clinique, que ce soit au niveau des critères d'inclusion ou des critères d'évaluation. Des marqueurs basés sur l'exploitation des acquisitions IRM sont désormais considérés régulièrement comme critères principaux d'évaluation. Dans le cadre d'études multicentriques en particulier, la gestion des acquisitions IRM doit considérer l'hétérogénéité des systèmes IRM en termes de constructeurs, de champs magnétiques, d'antennes de réception et de versions logicielles. Cela s'explique par le nombre de paramètres importants qu'il est possible de spécifier pour obtenir une acquisition IRM, la variabilité entre les solutions proposées par les constructeurs et les innovations techniques régulières.

Le REMI, le Réseau d'Entraide Multicentrique en IRM (https://remi.network/), a vu le jour en 2016 avec pour objectif de faciliter les contacts et l'entraide entre les acteurs académiques impliqués dans la recherche en et avec l'IRM. Il a reçu le soutien de la Société Française de Résonance Magnétique en Biologie et Médecine (SFRMBM), de FORCEi et le soutien financier de France Life Imaging (FLI) (1-3). Le réseau contribue notamment à faciliter la mise en place des protocoles IRM, en particulier multicentriques, grâce à un large partage de connaissances, de contacts, et de recommandations. Il a également pour but de favoriser la diffusion et l'intégration des nouveautés techniques dans la recherche clinique en IRM.

En 2019, Wiseman et al. ont proposé des recommandations pour réaliser des études longitudinales multicentriques en imagerie cérébrale. L'objectif du présent article est de préciser ce travail, en détaillant les spécificités à prendre en compte pour mettre en oeuvre et réaliser une étude en IRM quel que soit l'organe étudié, dans le contexte réglementaire français (4). En s'appuyant sur l'expérience et l'expertise des membres du REMI, nous proposons des recommandations détaillées, afin de mieux prendre en compte les spécificités de l'IRM à toutes 
les étapes de l'étude et de participer à l'amélioration de la qualité de la recherche en IRM. Ces recommandations prennent également en compte les spécificités des plateformes de recherche en IRM et des services d'imagerie clinique, ainsi que les contraintes réglementaires. La description d'une organisation idéale sera à moduler en fonction des possibilités en termes de ressources humaines et financières.

\section{Contexte et acteurs}

Selon la Circulaire DGOS/PF4 no 2011-329 du 29 juillet 2011 relative à l'organisation de la recherche clinique et de l'innovation et au renforcement des structures de recherche clinique, le développement de l'activité de recherche clinique dans les établissements de santé est essentiel pour la qualité et le progrès des soins, qu'il s'agisse de prise en charge diagnostique ou thérapeutique, et donc pour la performance du système de santé (5).

Lorsque qu'on s'intéresse plus spécifiquement aux intervenants dans la recherche clinique en France, il est important de distinguer :

1) les participants à la recherche, qu'il s'agisse de volontaires sains ou pathologiques,

2) le promoteur qui se définit comme étant la personne physique ou morale qui prend l'initiative et la responsabilité d'une recherche biomédicale sur l'être humain, qui en assure la gestion et vérifie que son financement est prévu selon l'article L.1121-1 du Code de la Santé Publique (6). Très souvent le rôle de promoteur est tenu par l'Inserm, le CNRS, le CHRU ou le CHU,

3) l'investigateur qui se définit par la personne physique qui dirige et surveille la réalisation de la recherche sur un lieu de recherche appelé centre investigateur ou site investigateur. Depuis la loi nº 2012-300 du 5 mars 2012 dite Loi Jardé - décret 2016- 
1537, le statut de cette personne est défini en fonction de la catégorie de la recherche et n'est désormais plus réservé aux médecins (7).

Au sein des établissements de santé, il convient d'identifier les structures ou organisations chargées d'appui à la recherche clinique. On distingue au sein de ces structures, une dizaine de métiers spécifiques à la recherche clinique. On y retrouve :

- les Délégations à la Recherche Clinique et à l'Innovation (DRCI) pour l'organisation, l'administration, la gestion, le contrôle, l'appui technico-réglementaire de la recherche clinique. Pour cela, les DRCI doivent assurer un rôle d'information, d'incitation auprès des équipes hospitalo-universitaires et de tous les investigateurs qui souhaitent mener un projet. Ces rôles sont assurés par des chefs de projets de recherche clinique, des Attachés de Recherche Clinique (ARC), et spécialistes en vigilance hospitalière ;

- les centres d'investigation clinique (CIC) et les centres de recherche clinique (CRC) permettant le soutien spécifique pour l'aide au montage, à l'inclusion et à l'investigation en recherche clinique. Ces structures intègrent notamment :

- des Coordonnateurs d'Études Cliniques (CEC), des Techniciens d'Etude Clinique (TEC), des Infirmières de Recherche Clinique (IRC), des Manipulateurs en ElectroRadiologie Médicale (MERM), et parfois des ingénieurs et/ou physiciens spécialisés en IRM pour l'aide à l'investigation et à l'acquisition des données.

- des méthodologistes, statisticiens, gestionnaires de données biomédicales, ingénieurs en recherche clinique pour le support méthodologique, les biostatistiques, la gestion et le traitement des données.

Des plateformes de recherche en imagerie peuvent également proposer un service d'accompagnement des investigateurs pour l'acquisition et le traitement des données en IRM. Elles peuvent être portées par des CHU ou des Établissements publics à caractère scientifique 
et technologique (EPST) parmi lesquels le CNRS, l'Inserm, l'Université, Inrae et Inria. Les personnels de ces plateformes peuvent être issus des établissements de santé ou des instituts de recherche - ingénieur de recherche, ingénieur d'études, assistant ingénieur, technicien de recherche et de formation, adjoint technique de recherche et de formation.

Depuis la Loi Jardé, un Comité de Protection des Personnes (CPP) doit être consulté pour tout type de recherche prospective, interventionnelle et non interventionnelle, dès lors que le projet porte sur une recherche impliquant la personne humaine. Il se prononce sur les conditions dans lesquelles le promoteur de la recherche assure la protection des personnes et notamment des participants, sur le bien-fondé, la pertinence du projet de recherche et sur sa qualité méthodologique. L'Agence Nationale de Sécurité du Médicament et des produits de santé (ANSM) est quant à elle l'autorité compétente en matière de vigilance. Elle veille à la sécurité de l'emploi des médicaments et contribue à leur bon usage. Elle coordonne et assure également la mise en œuvre du système national de pharmacovigilance. La Commission Nationale de l'Informatique et des Libertés (CNIL) est notamment chargée de veiller à la protection des données personnelles des participants inclus dans les études. Selon la définition de la CNIL, ces données peuvent être contenues dans les fichiers et traitements informatiques ou papiers, aussi bien publics que privés.

\section{Montage de projet}

\subsection{Conception du projet}

Le déroulement d'un projet de recherche clinique comprend les étapes illustrées Figure $1:$ le montage du projet et la recherche de financement, les soumissions règlementaires, le recueil des données, l'analyse des données et la valorisation des résultats. 


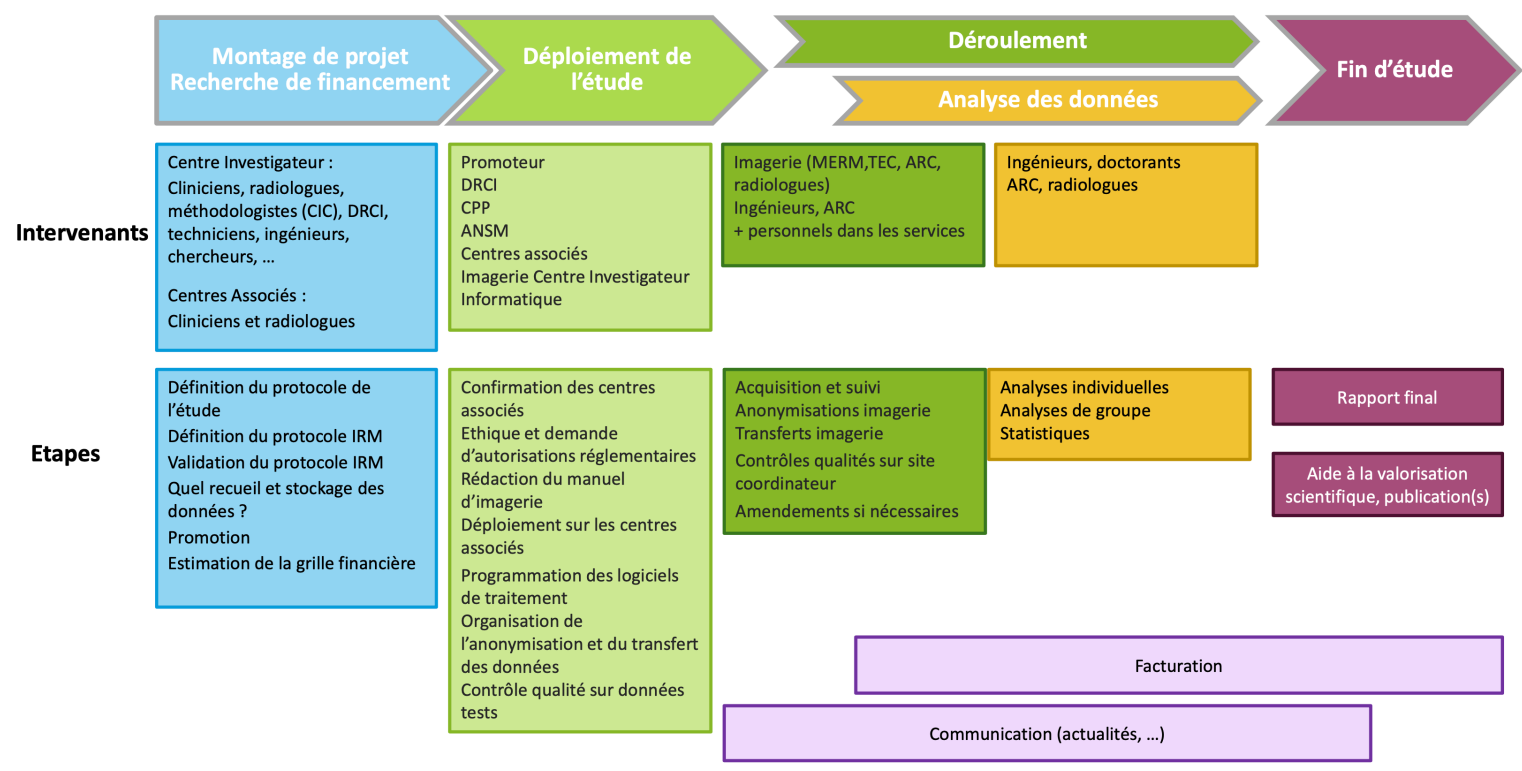

Figure 1 : Déroulement d'un projet de recherche clinique

La préparation spécifique à l'imagerie comprend quant à elle : l'élaboration du protocole IRM de l'étude qui détermine les données à recueillir, la définition du plan d'analyse et de gestion des données, l'évaluation des ressources humaines et matérielles - actes, matériels spécifiques - nécessaires ainsi que le coût associé. Ces étapes permettent d'étayer la demande de financement, d'évaluer la faisabilité de l'étude et de préparer sa réalisation.

Tout en s'appuyant sur les données de la littérature, il est utile de prendre en compte les spécificités techniques des équipements et d'associer les différents acteurs dès les premières étapes du projet. En effet, certaines données peuvent se révéler inexploitables si le protocole d'imagerie n'est pas défini précisément, harmonisé et testé en amont de l'inclusion des participants.

La Figure 2 reprend tous les intervenants de la recherche, au sein du centre coordonnateur qui aura en charge la coordination globale, et des centres associés à l'étude. 


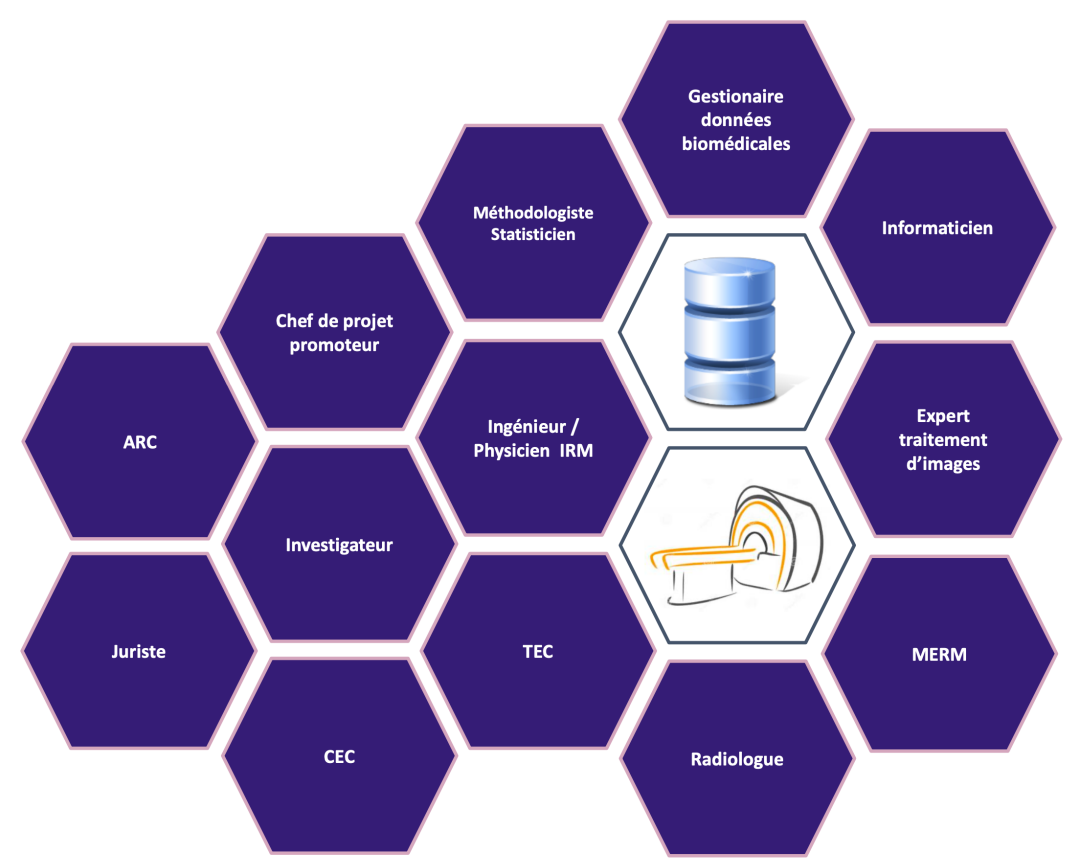

Figure 2 : Exemple d'organisation des intervenants d'une étude de recherche clinique (CdP :

Chef de projet, CEC : Coordonnateurs d'Études Cliniques, TEC : Techniciens d'Etude

Clinique, ARC : attachés de Recherche Clinique, MERM : Manipulateurs en Electro-Radiologie

\section{Médicale)}

Les référents IRM que sont les radiologues, MERM, TECs, ingénieurs et/ou physiciens IRM seront en charge de la mise en place des acquisitions, leur réalisation, le transfert des données acquises et le cas échéant de la relecture des images. La collecte, la gestion et l'analyse des données par le centre coordonnateur peuvent être confiées à des informaticiens qui déterminent les solutions techniques à utiliser pour recueillir les données, les structurer, les conserver, en contrôler la qualité, et les analyser.

\subsection{Définition du protocole IRM et faisabilité locale}

La définition du protocole IRM doit se faire en accord avec l'équipe IRM pour s'assurer de l'adéquation entre les objectifs de l'étude, les critères d'évaluation, le protocole IRM et sa faisabilité. L'IRM peut constituer l'objet de la recherche ou être un produit associé à l'étude. 
De plus, les contraintes seront différentes selon que l'étude utilise des données recueillies dans le cadre de la pratique habituelle ou hors soins courants. En effet, les explorations en soins courants suivent les recommandations des sociétés savantes telles que celles de la Société Française de Radiologie (SFR) ou celles de la Société Européenne de Radiologie (ESR) $(8,9)$. Les séquences de recherche peuvent être conventionnelles, à savoir réalisables dans le cadre de la pratique habituelle sans contraintes spécifiques, ou avancées, demandant des options logicielles spécifiques ou des consignes de réalisation potentiellement plus complexes. Une optimisation spécifique de certaines séquences pourra être envisagée, compromis entre la durée d'acquisition, la géométrie (couverture, résolution spatiale), le contraste et le rapport signal à bruit nécessaires permettant de répondre à l'objectif de l'étude, sans oublier la robustesse au traitement des données qui sera réalisé. Les tenants et aboutissants de cette optimisation sont illustrés en Figure 3.

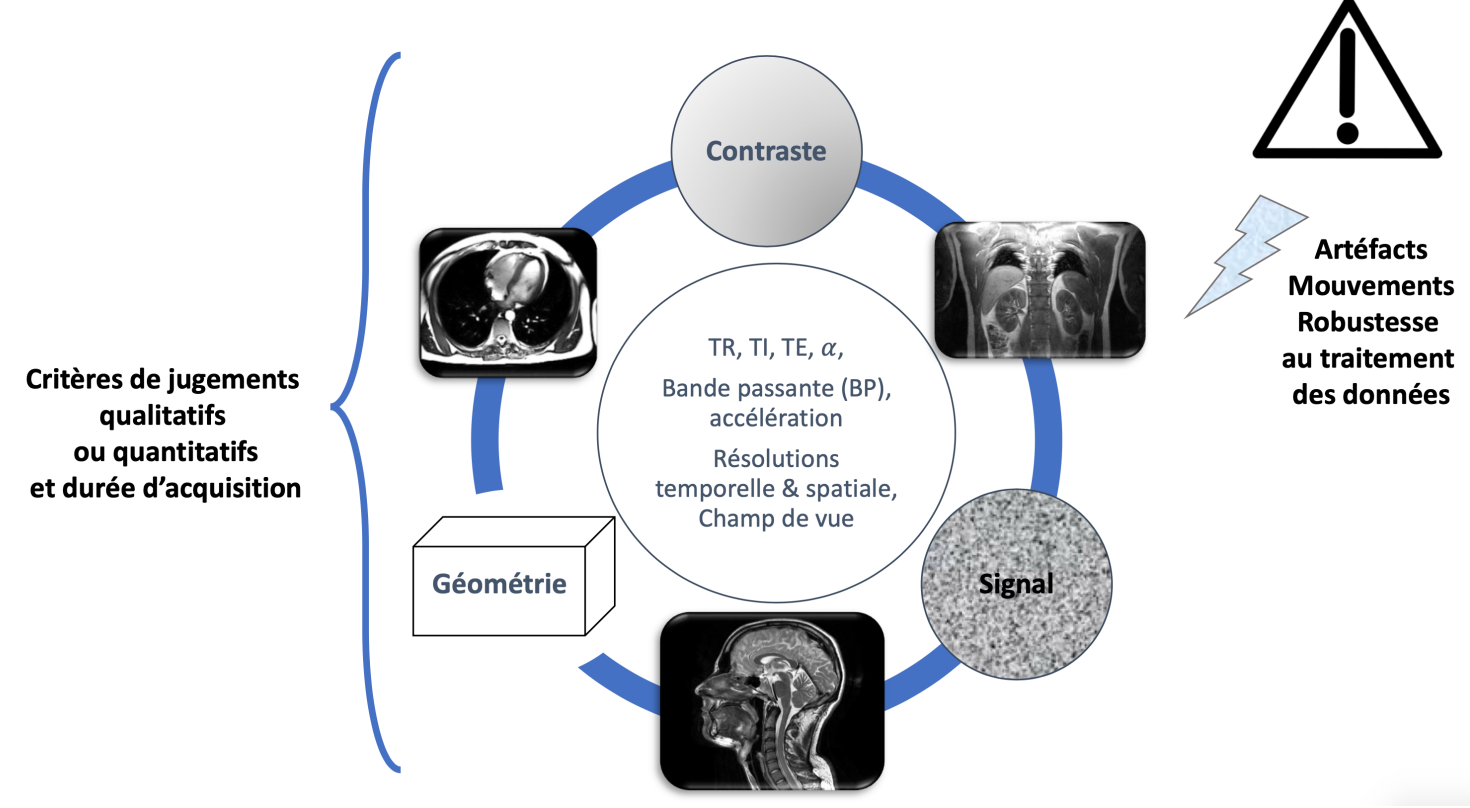

Figure 3 : Optimisation de séquences en IRM - Temps de Répétition (TR), Temps d'Echo (TE), Temps d'Inversion (TI), angle de bascule ( $\alpha$ ), Bande Passante (BP), accélération, résolutions temporelle et spatiale, champ de vue ... 
La définition du protocole IRM doit également prendre en compte la capacité des participants à respecter les contraintes imposées par l'IRM, comme le fait de demeurer immobile pendant l'ensemble des séquences d'acquisition, de réaliser certaines tâches - cognitives ou respiratoires - et de venir à l'ensemble des visites prévues par l'étude. Le protocole peut être scindé entre une partie "obligatoire" pour les objectifs principaux de l'étude et une partie “optionnelle", selon les possibilités des centres pour les objectifs secondaires de l'étude. Les séquences devront être listées dans le protocole de l'étude à partir des noms génériques, tous constructeurs confondus. Il n'est pas nécessaire de donner le détail des paramètres mais il convient de préciser la durée totale de l'examen.

Si une séquence d'acquisition IRM est nécessaire pour répondre à l'objectif principal de l'étude, il sera nécessaire en cas de données non conformes - non-respect du protocole, problème technique ou mouvement du participant - de la répéter lors de la même session d'acquisition ou d'une nouvelle session d'acquisition. Ce cas devra être prévu réglementairement et financièrement.

Nous recommandons d'acquérir le protocole IRM établi en version préliminaire sur un objettest et/ou sur un participant. L'analyse de ces données permettra de vérifier qu'elles sont conformes aux attentes et idéalement de mettre en place les outils de contrôle qualité ou de traitement d'images et les ressources matérielles et humaines nécessaires à leur exploitation. L'acquisition de données sur objet-test peut servir d'échantillon de référence au moment de l'acquisition sur participant, et être une référence de la stabilité de l'imageur tout au long de l'étude en particulier lorsque des séquences quantitatives sont réalisées. 


\subsection{Faisabilité du protocole IRM dans le cadre multicentrique}

Un questionnaire, organisationnel et technique, adressé aux centres associés permet notamment d'estimer le potentiel d'inclusion, de s'assurer de l'intérêt des centres pour l'étude, de leur adhésion au protocole, de sa faisabilité, d'identifier les contacts opérationnels et la disponibilité de créneaux pour la recherche. Un volet technique du questionnaire permettra de recueillir sur chaque site participant le champ magnétique, le modèle et la version logicielle de l'IRM sur laquelle sera réalisée l'étude, la planification d'une mise à jour de l'IRM ou du logiciel d'acquisition pendant la durée de l'étude, les antennes, objets-tests et options disponibles.

Les caractéristiques techniques précises de chaque séquence dans le cadre de l'étude pourront ainsi être harmonisées autant que possible entre les centres, afin d'aboutir à des données d'imagerie réalisables et homogènes. La Figure 4 illustre ce travail d'harmonisation multicentrique des protocoles IRMs pour obtenir des images comparables, radiologiquement et quantitativement selon les sites, les constructeurs, modèles, champs magnétiques et versions logicielles des systèmes IRMs.

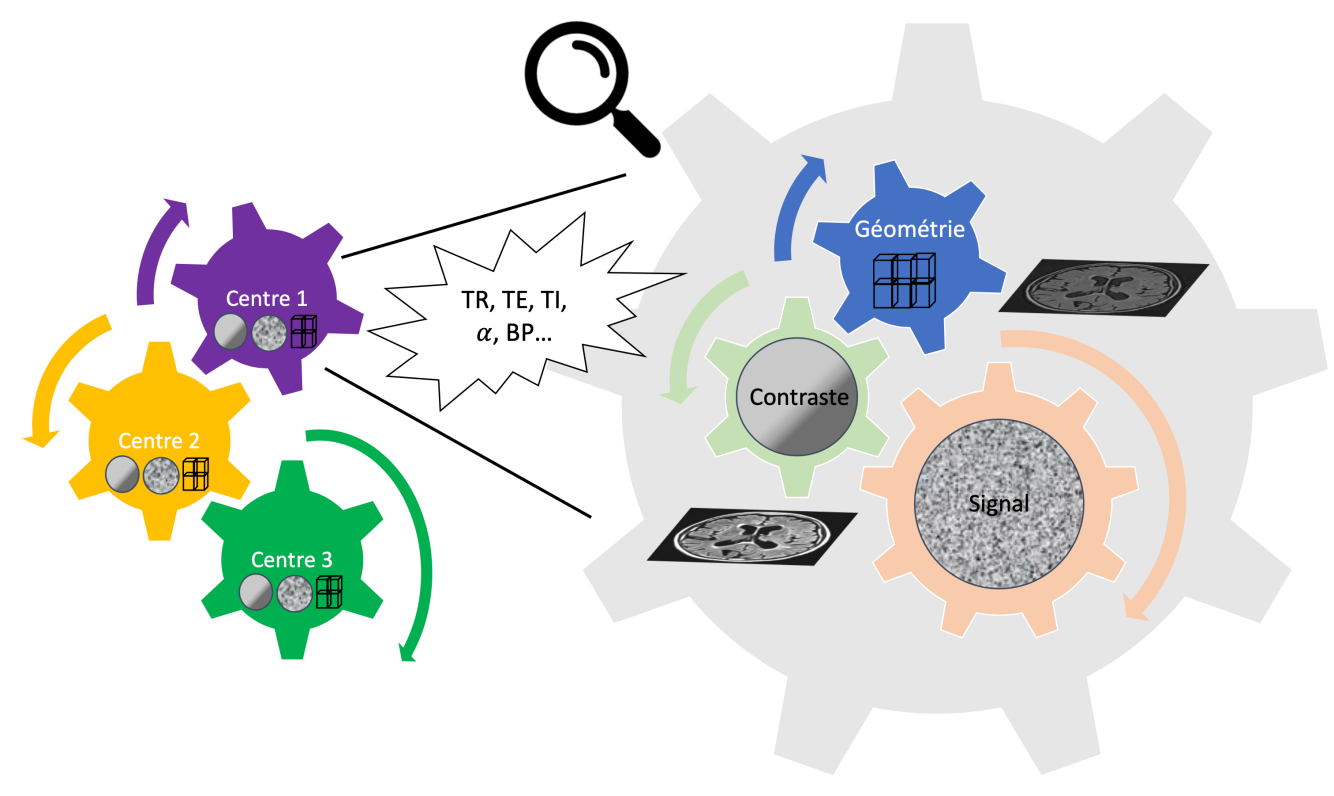


Figure 4 : Harmonisation multicentrique de protocoles IRMs pour obtenir des images comparables, radiologiquement et quantitativement

Si le protocole doit être harmonisé sur des IRMs de différents constructeurs, un temps supplémentaire dédié à l'adaptation du protocole IRM par une ressource locale ou une structure dédiée doit être prévu et inclus dans la grille budgétaire ainsi que dans le calendrier de l'étude. En effet, le schéma de la séquence IRM - chronogramme et reconstruction, les noms et valeurs des paramètres diffèrent selon les constructeurs et l'harmonisation du protocole entre les différents systèmes IRMs, pour rendre les données les plus comparables possibles, sera facilitée s'il est possible de déterminer parmi les centres participants un centre référent par type de constructeur. Celui-ci deviendra un partenaire privilégié de l'étude pour évaluer l'adaptabilité des séquences et des paramètres sur son IRM. Il est préférable que ces centres référents aient des créneaux IRM facilement accessibles et une autorisation éthique locale pour réaliser des tests sur participants. Enfin, lors de la phase de déploiement, les protocoles IRM seront à finaliser et à décliner par type de machine, version, configuration, spécificités techniques et options logicielles. Certaines études prévoient le passage d'un même sujet témoin dans les différents centres mais cela est compliqué à réaliser si le nombre de centres est important.

Si des acquisitions de qualification du protocole IRM sont prévues dans les centres associés sur un volontaire, sain ou pathologique, il convient de les inclure dans le protocole de l'étude et de les budgétiser. Un consentement spécifique pour cette qualification et une indemnité pour le sujet devront être envisagés. En l'absence d'accord éthique, certaines acquisitions pourront être effectuées sur objet-test pour valider la faisabilité technique, à budgétiser également.

Pour la certification du système, les acquisitions sur objet-test permettent de valider les performances de l'IRM. Pour la qualification du centre, lors de la mise en place du protocole 
IRM, les acquisitions sur objet-test permettent de vérifier la conformité du protocole IRM et d'acquérir des données de référence. Réalisées régulièrement au cours de l'étude, ces acquisitions permettent de vérifier la stabilité de l'imageur. En particulier, lorsque des mesures quantitatives sont prévues, le contrôle qualité machine sur objet-test est recommandé pour évaluer la cohérence des valeurs obtenues et prendre en compte la variabilité entre les différents centres. L'objet test peut être un objet commercialisé - ACR, Spin Safety, Magphan, NIST notamment - auquel est associé une procédure de contrôle qualité permettant de quantifier différents paramètres géométriques et de signal (10-13). Cet objet-test peut également provenir d'un développement local pour la recherche avec des caractéristiques maîtrisées.

\subsection{Collecte des données}

La solution technique de centralisation des données doit prendre en compte la sécurité des données lors du transfert, la facilité d'utilisation et les possibilités d'installation de l'outil localement notamment en termes de compatibilité avec les procédures de sécurité informatiques des centres participants, le format des données recueillies - Digital Imaging and Communication in Medicine (DICOM) le plus souvent - et les conditions de stockage (14). Plusieurs solutions existent, et des structures académiques proposent des services d'archivage et de traitement de données. Le mode de centralisation des données retenu est à décrire dans le protocole de l'étude et à intégrer dans la grille budgétaire.

\subsubsection{Dé-identification ou pseudonymisation}

Les données d'imagerie sont à considérer comme des données à caractère personnel au sens du Règlement Général sur la Protection des Données (RGPD), et non comme des données anonymisées (15). En effet, il reste possible d'isoler un individu, de relier entre eux des enregistrements relatifs à un individu ou de déduire des informations concernant un individu 
conformément à l'avis du Comité Européen de la Protection des Données (CEDP). Des précautions sont à prendre et un plan d'analyse des risques à réaliser pour encadrer la circulation des données au sein de l'étude.

En pratique, les données recueillies dans le cadre d'une étude sont pseudonymisées avec le numéro du sujet dans l'étude. Toute information directement identifiante à partir des données d'imagerie acquises telles que nom, date de naissance complète, adresse du site investigateur, prescripteur, radiologue est à supprimer. Le standard DICOM donne des recommandations pour la pseudonymisation. Des outils libres, commerciaux sur l'imageur, sur le Picture Archiving and Communication System (PACS) ou indépendants permettent de dé-identifier les données DICOM tels que DICOM Anonymizer ou DICOM Cleaner (16,17). Sur certains outils, le paramétrage des différents champs DICOM à supprimer n'est pas possible. Le développement d'un outil ad-hoc nécessite de définir les champs à conserver - liste blanche ou à l'inverse les champs à supprimer - liste noire - en s'assurant que cette pseudonymisation permette le traitement des données. A titre d'exemple de pseudonymisation excessive, nous pouvons citer les valeurs de $b$ et directions pour les données de diffusion qui peuvent disparaître lors d'un transfert via le PACS.

Il est à noter que certains champs privés peuvent être utilisés différemment selon les constructeurs, et que des informations peuvent également être présentes dans le fichier DICOM, non accessibles aux lecteurs de métadonnées DICOM mais accessibles via un éditeur de texte.

\subsubsection{Centralisation des données}

La centralisation des données au fur et à mesure des acquisitions permet de minimiser le risque de perte de données. Il est préférable d'opter pour une solution virtuelle tel qu'un serveur sftp ou équivalent, économique et rapide, plus fiable dans le temps qu'un stockage sur 
support externe. Cette centralisation rend possible un contrôle visuel ou automatisé de la qualité des données à réception, qui permet d'informer le centre de la conformité de l'examen et, le cas échéant, de proposer des mesures correctives pour les examens suivants, ainsi qu'une potentielle répétition de l'examen. Des outils ad hoc peuvent être développés ou des solutions open-source collaboratives utilisées (18). Ce contrôle à réception permet également de renseigner l'investigateur principal sur une estimation du nombre de sujets exploitables.

\subsubsection{Stockage et accessibilité des données}

L'estimation de la volumétrie d'un examen permet de prévoir l'espace de stockage nécessaire et le coût associé. Le cadre de conservation (pendant la durée de l'étude), d'archivage (à la fin de l'étude) et d'accès aux données de façon compatible avec les contraintes de la CNIL devra être défini (accès sécurisés, extraction de données en fin d'étude ou à intervalles réguliers pour pré-traiter les données d'imagerie ou permettre des analyses intermédiaires).

\subsection{Estimation de la grille budgétaire}

Le Comité National de Coordination de la Recherche (CNCR) a publié une grille des surcoûts pour les études pharmaceutiques industrielles et il en existe une variante pour les études académiques (19). Les principaux postes liés à l'IRM sont décrits ci-dessous.

Pour le centre coordonnateur, du temps personnel (ARC, TEC, MERM, Ingénieur, Informaticien), est à budgétiser pour :

- la mise au point du protocole IRM localement,

- l'harmonisation du protocole IRM,

- la mise au point de la solution de transfert de données,

- la gestion et le stockage/archivage des données,

- le déploiement du protocole IRM et de la solution de transfert en multicentrique,

- la mise au point des outils de contrôle qualité, 
- le contrôle qualité des données à réception,

- la mise au point des outils d'analyse de données,

- l'analyse des données,

- la lecture centralisée le cas échéant.

Dans chaque centre de l'étude :

- le forfait de mise en place en IRM - prise de connaissance du protocole, réponse aux questionnaires; estimation des surcoûts IRM; formation virtuelle ou présentielle à l'étude; installation du protocole sur l'IRM et itérations si nécessaire pour sa validation; participation à la réunion de mise en place,

- la qualification IRM du centre - réalisation et validation d'une acquisition de certification ou dummy run sur objet test ou sur volontaire, sain ou pathologique,

- le coût/surcoût par examen IRM sur volontaire, sain ou pathologique,

- le coût/surcoût par examen pour les consommables,

- le surcoût éventuel par examen IRM sur objet-test (contrôle qualité durant l'étude),

- le compte-rendu médical (contenu attendu et coût associé),

- la dé-identification des données (temps humain),

- le transfert des données (temps humain),

- la réponse aux requêtes du promoteur (temps humain).

Parmi les centres associés, nous pouvons distinguer les centres référents qui sont des centres où les tests d'ajustements de protocoles IRM se feront. Pour ces centres référents, du temps supplémentaire pourra être ajouté pour les ajustements d'harmonisation du protocole IRM. 


\section{Déploiement de l'étude}

\subsection{Dépôts réglementaires}

Une fois le financement obtenu, les investigateurs sont sollicités pour confirmer leur volonté et possibilité de participer à l'étude, mettre à jour les informations collectées lors de la phase de montage de projet présentées au paragraphe 2.3. L'harmonisation finale du protocole IRM est réalisée à ce stade.

La liste des centres participants ainsi que le protocole de l'étude seront transmis au promoteur qui soumettra l'étude au CPP, à l'ANSM et, si nécessaire, à la CNIL.

Après obtention de l'accord du CPP et de l'autorisation ANSM, l'étude sera mise en place réglementairement dans le centre coordonnateur puis dans l'ensemble des centres une fois les conventions signées entre centres.

\subsection{Documents support}

L'instruction par le CPP peut prendre plusieurs mois. Ce temps peut être mis à profit pour finaliser les documents support, en particulier le manuel pour l'acquisition IRM, la partie IRM du cahier d'observation papier ou électronique (respectivement nommé CRF/e-CRF) et la feuille de transmission IRM.

Le manuel d'imagerie doit être succinct et préciser notamment :

- l'objectif de l'étude ;

- le matériel à utiliser : antenne, capteurs, produit de contraste, injecteur, instrumentation connexe...;

- le positionnement du participant : décubitus ventral ou dorsal, positionnement des membres ;

- l'utilisation d'une référence externe si besoin : marqueur local indiquant la latéralité, objet-test aux concentrations spécifiques...; 
- les consignes à donner au participant : ne pas bouger pendant l'examen et des consignes plus spécifiques en cas de resting-state ou d'IRM fonctionnelle, d'IRM cardiaque (apnée), d'IRM hépatique (respiration calme)...;

- les séquences à réaliser avec les paramètres recommandés selon le modèle d'IRM ;

- l'ordre des séquences, celles qui sont essentielles et l'importance de faire les acquisitions en suivant toujours le même protocole IRM avec le même matériel ;

- les consignes sur le champ de vue, notamment la couverture demandée et le positionnement du bloc de coupes selon un repère anatomique et leur orientation axial strict, coronal, sagittal, etc... ou la possibilité de suivre le positionnement automatique des constructeurs ;

- les paramètres à vérifier avant le lancement des séquences (volume et qualité du shim, ajustement de fréquence en spectroscopie notamment ou pour améliorer la saturation de graisse, sens de la phase);

- les éventuelles adaptations à faire sans changer les paramètres d'acquisition (usersCVs, mode avancé, enregistrement des données physiologiques, gestion du dépôt d'énergie spécifique ou SAR en anglais pour Specific Absorption Rate);

- les reconstructions d'images nécessaires (anatomiques sur trois plans, soustractions, cartes paramétriques, autres paramètres de reconstructions...);

- les instructions en cas de participant agité ou douloureux, si des séquences sont à recommencer par exemple ou s'il peut être acceptable de modifier les paramètres afin de diminuer le temps d'acquisition ;

- la procédure de pseudonymisation ;

- les instructions pour le transfert de données ;

Ce manuel d'imagerie peut être adapté selon les centres et les constructeurs suite à la définition du protocole IRM réalisée en amont par le centre référent IRM. Pour certains 
systèmes, il est possible de fournir avec ce manuel un fichier contenant le protocole IRM qui peut être importé directement dans le système IRM.

Une fiche de transfert de données permet de tracer la date d'acquisition et de transfert, et peut comprendre les informations sur les séquences acquises et les problèmes rencontrés lors de l'acquisition, le type de produit de contraste éventuellement utilisé ainsi que la quantité injectée et l'heure de l'injection.

La partie IRM du CRF/e-CRF comprend uniquement la date de réalisation de l'examen et de transmission des données. Les mesures radiologiques ou quantitatives issues de l'IRM ne seront peut-être disponibles qu'ultérieurement après traitement des images et ne peuvent pas être intégrées dans les champs du CRF/e-CRF.

\subsection{Implémentation du protocole IRM}

En parallèle de la signature de la convention financière, tous les interlocuteurs IRM du centre (radiologues, MERMs, TECs, ingénieur ou physicien IRM) doivent être informés par le centre coordonnateur et/ou en interne pour mettre en place le protocole IRM.

Les paramètres des séquences saisis peuvent être exportés et transmis (format pdf, txt ou constructeur) au centre coordonnateur pour vérification. Une fois les paramètres validés, l'examen de qualification ou "dummy run" pourra être réalisé sur objet-test et/ou sur sujet puis les données seront envoyées au centre coordonnateur via la solution de transfert choisie. Cette acquisition permet également de tester les différentes étapes du flux de données, de l'acquisition au transfert des données et d'identifier d'éventuels problèmes à résoudre en amont du démarrage de l'étude.

\subsection{Mise en place réglementaire}

La réunion de la mise en place organisée par le promoteur marque le démarrage possible des inclusions. Il est indispensable que cette réunion associe les acteurs locaux du centre 
d'imagerie pour s'assurer que les circuits nécessaires sont en place, et qu'ainsi le délai entre la mise en place réglementaire et la première inclusion soit le plus court possible.

Cette réunion permet de préciser les circuits de demande de rendez-vous et les contraintes spécifiques des différents acteurs, de vérifier que les demandes IRM pour l'étude seront clairement identifiées par le centre IRM afin d'assurer que les acquisitions seront réalisées avec le protocole IRM validé. La première acquisition de chaque centre devrait être vérifiée avec soin, en particulier si l'examen de qualification n'a pu être réalisé que sur un objet-test.

\section{Conduite de l'étude}

\subsection{Acquisitions}

Tout comme le radiologue, l'ingénieur et/ou physicien IRM, les MERMs ont un rôle primordial dans l'étude. Leur implication dans l'étude et leur rigueur pour appliquer les consignes décrites dans le manuel d'acquisition IRM seront un atout pour l'obtention de données de bonne qualité et homogènes (20).

La phase d'acquisition des données, qui peut durer plusieurs années, doit faire l'objet d'un suivi constant, notamment pour :

- les feuilles de transmission,

- le CRF/e-CRF,

- la vérification du bon déroulement du protocole et du respect des consignes,

- la dé-identification,

- le transfert des données,

- leur réception par le centre coordonnateur.

Ces documents seront contrôlés en cas d'audit. 


\subsection{Changement d'IRM en cours d'étude}

En cas de prévision de changement de système IRM ou de mise à jour majeure sur ce système, il convient de prévenir l'investigateur principal du centre concerné ainsi que le référent IRM du centre coordonnateur. Il n'y a pas de recommandation unique, il faut aviser au cas par cas en fonction des pré-requis de l'étude et du nombre de sujets restant à inclure. Outre la qualification du nouveau système IRM, un nouveau travail d'harmonisation du protocole IRM sera nécessaire si le modèle d'IRM n'est pas connu dans le cadre de l'étude.

Dans le cas d'une étude transversale multicentrique, le changement d'IRM peut être considéré comme l'ajout d'un nouveau centre et avoir un impact peu préjudiciable sur l'homogénéité des données IRM acquises.

Cela peut être plus problématique dans le cas d'une étude longitudinale. La programmation des examens IRM peut être adaptée (suivis anticipés ou retardés) pour s'assurer de pouvoir colliger les données. Le centre coordonnateur pourra également demander la réalisation d'un examen sur volontaire sur l'ancien et le nouveau système IRM afin de vérifier la comparabilité des images obtenues ainsi que des mesures extraites. Bien que difficiles à anticiper lors du montage de l'étude, ces cas doivent être prévus dans le protocole de l'étude et dans la grille budgétaire.

\subsection{Conformité des données}

Le contrôle visuel de la qualité des données dans chaque centre repose en premier lieu sur le MERM et le radiologue local référent de l'examen pour les images conventionnelles. Dans le cas de protocole de recherche, le protocole d'acquisition IRM doit respecter les recommandations du manuel d'imagerie et ne doit pas être modifié sans accord du centre coordonnateur. 
Le contrôle centralisé de la conformité des données par le centre coordonnateur a pour but de vérifier le respect du protocole IRM tel que détaillé dans le manuel d'imagerie et l'intégrité des données reçues. Réalisé au fur et à mesure, il permet de revenir vers les centres pour apporter des mesures correctives..

La première étape du contrôle consiste à vérifier les séquences du protocole IRM (ordre, nommage, nombre de fichiers reçus, doublons), les paramètres d'acquisition, le matériel utilisé (système et antenne utilisés, version logicielle), la non présence d'informations DICOM identifiantes ou a contrario la présence d'informations indispensables à l'analyse des données. Il peut être facilement automatisé à partir des champs DICOM, sous réserve que les champs nécessaires aient bien été conservés.

La seconde étape concerne le contenu des images et vise à caractériser la qualité globale de chaque examen, à déterminer si le système d'acquisition se comporte normalement et si les données sont exploitables. Au-delà du contrôle visuel systématique, des outils disponibles pour la communauté existent et des développements spécifiques peuvent être réalisés, afin d'extraire des indices quantitatifs ou qualitatifs. Ils peuvent alors être utilisés pour préciser la qualité des examens et éventuellement exclure des données. Cette étape de contrôle qualité peut être envisagée sur des données IRM déjà prétraitées ou peut également comprendre des étapes de prétraitements qui seront détaillés ci-après. Par souci de clarté, nous distinguerons les étapes de prétraitements (corrections de mouvement, d'intensité, de distorsions, d'artefacts, de bruit, recalage vers un espace standard ou entre points de temps...), du traitement des données (transformation en cartes paramétriques (diffusion, relaxométrie, perfusion, ...) ou statistiques, sélection des régions d'intérêts manuelle ou automatique (segmentation) et de l'analyse des données.

Des informations non DICOM (données brutes dans l'espace fréquentiel, données physiologiques, protocole d'acquisition ou de stimulation, réponses du participant durant 
l'examen IRM) peuvent également être recueillies. Dans la plupart des cas, il s'agit de s'assurer de la présence de ces informations. Dans le cas des données de spectroscopie IRM, la résolution spectrale et le rapport signal sur bruit doivent être vérifiés visuellement à défaut d'outils dédiés.

A la fin de l'étude ou lors des analyses intermédiaires, le contrôle centralisé est un préalable au traitement et à l'analyse des données, donnant une information essentielle sur la fiabilité des résultats qui seront obtenus à partir des données.

\subsection{Analyse des données}

L'analyse des données permet d'obtenir les mesures nécessaires pour l'évaluation des critères de jugement de l'étude. C'est une phase chronophage, difficilement automatisable dans sa totalité et à intégrer dans l'estimation budgétaire et dans le calendrier. Le plus souvent réalisée par le centre coordonnateur, il est aussi possible d'envisager dans ces études multicentriques un partage des objectifs d'analyse d'images au sein du consortium.

Les données IRM sont complexes, elles intègrent à la fois des informations spatiales, fréquentielles, et temporelles. Le plus souvent, ce sont des images mais des données non image (spectres, données physiologiques entre autres) peuvent également être recueillies. Le traitement de ces données nécessite l'utilisation d'outils dédiés (logiciels commercialisés ou logiciels libres collaboratifs) et de faire appel à des compétences et à une expertise spécifique en traitement du signal (outils développés pour les besoins spécifiques de l'étude).

Afin d'éviter d'introduire un biais dans les résultats, le traitement des données s'effectue en aveugle des caractéristiques du participant (maladie, groupe expérimental) selon le design de l'étude. De même, s'il y a une lecture centralisée, elle doit être réalisée par des lecteurs 
indépendants de l'étude, formés et qualifiés par le radiologue référent de l'étude. L'extraction d'une ou plusieurs région(s) d'intérêt(s) (ROI) pour le calcul des mesures d'intérêt peut être faite manuellement par un opérateur expert ou automatiquement par des outils dédiés (outils logiciels validés et commercialisés, disponibles dans un environnement de recherche ou réalisés localement et validés dans un cadre similaire). Ces étapes sont à détailler dans une procédure complète, validée par l'investigateur principal. Dans le cas d'une lecture centralisée ou d'une extraction manuelle, le lecteur devra être tracé et la reproductibilité intra et inter observateur devra être évaluée.

Les calendriers du traitement (au fur et à mesure, échéances intermédiaires ou fin d'étude) et de l'analyse des données IRM (échéances intermédiaires, fin d'étude), doivent être en conformité avec le protocole validé par les autorités compétentes. Si le traitement est réalisé au fur et à mesure de la réception des données ou lors d'analyses intermédiaires, il est nécessaire de gérer de façon précise les versions des environnements et logiciels utilisés. Cela permettra de prendre en compte la variabilité analytique pour contrôler leur influence sur les résultats. Des méthodes telles que des tests de non régression permettent d'évaluer l'effet d'un changement d'un des éléments de la chaîne de traitement et la nécessité de refaire ces traitements en préalable à l'analyse finale des données.

La fiabilité des résultats des analyses devrait être évaluée par un contrôle qualité adapté à la taille de l'échantillon. Une analyse de la variabilité inter-sujets, intra-centre, inter-centres peut venir appuyer ce travail de contrôle qualité.

En outre, la stratégie face aux données non fiables (à cause d'un échec au contrôle qualité de données brutes ou de traitement) devrait être évoquée dès le montage de l'étude (données 
manquantes, exclusion du jeu de données complet, adaptation des paramètres des méthodes utilisées...).

Les informations ainsi obtenues seront importées soit dans le CRF/e-CRF de l'étude soit dans une base de données dédiée aux résultats IRM comprenant les résultats du contrôle qualité, versions logicielles, systèmes et lecteurs. L'extraction du CRF/e-CRF après gel de la base de données et/ou la base de données des résultats IRM seront fournis au statisticien de l'étude.

\section{Conclusion}

La réalisation d'une étude multicentrique en IRM repose sur plusieurs étapes clés : le montage de l'étude comprenant la définition du protocole IRM et sa faisabilité en multicentrique, l'identification des ressources matérielles et humaines nécessaires ainsi que le coût associé, la collecte des données, le contrôle de leur conformité, le traitement et l'analyse des données. Tous les acteurs doivent être associés dès le montage du projet. Aux étapes clés détaillées dans cet article doivent s'ajouter des étapes qui ont fait l'objet d'études d'autres équipes, traitant notamment du fléchage et de la facturation des examens, de la gestion technique et règlementaire des données, de la compliance du sujet et de la fréquence d'artéfacts de mouvements dans les images $(21,4,22)$.

La Haute Autorité de Santé s'est intéressée à l'établissement d'indicateurs pour l'amélioration de la qualité et de la sécurité des soins évaluant la conformité des demandes d'examens d'imagerie (CDEI). Ces indicateurs étaient administratifs (date de la demande, service demandeur, nom du médecin demandeur, identité du patient, date de naissance du patient) et cliniques (région anatomique, motif de l'examen, finalité). Les campagnes de 2012 et 2014 
ont montré que ce travail avait permis d'augmenter le taux de conformité des demandes dans $26 \%$ des 91 établissements ayant participé $(23,24)$.

Toutefois, il n'existe pas à notre connaissance d'études s'intéressant à la conformité des données d'imagerie issues des protocoles de recherche. Outre les recommandations pratiques d'organisation présentées dans cet article, nous recommandons de bien sélectionner les centres pour éviter notamment d'avoir des systèmes IRM sur lesquels ont été acquis un faible nombre de jeux de données, et maîtriser l'effet système IRM dans les données d'imagerie. Ces recommandations rejoignent les conclusions du rapport publié en 2013 par le Centre National de Gestion des Essais de Produits de Santé (CeNGEPS) (25).

En 2013 également, Cornu et al ont évalué les principaux écueils des essais cliniques parmi lesquels la conception, les procédures, la gestion des données et l'analyse (26). L'impact sur les résultats est presque toujours une surestimation de l'effet traitement. Un monitoring important, ciblé sur les risques d'erreurs, avec des méthodes diversifiées est préconisé par les auteurs. En 2018, une session du congrès européen de radiologie était dédiée au développement de la recherche clinique dans les services d'imagerie. Elle comprenait une présentation sur le management de la recherche en imagerie et une présentation sur l'importance du contrôle qualité machine et données dans le cadre de la recherche en imagerie (qui plus est multicentrique et quantitative) avec la recherche de biomarqueurs (27-29). Le caractère clé de l'implication du MERM dans les projets de recherche, au-delà de la réalisation des images, a également été développé lors de cette session faisant écho à la création de diplômes universitaires dédiés à la formation de MERM en recherche clinique (30). La recherche clinique et paramédicale a également été mise à l'honneur dans l'édition de la Revue du Manipulateur en 2012 (21). De plus, l'expertise technique de physiciens IRM, ingénieurs, informaticiens et traiteurs d'images est également nécessaire pour maîtriser la conduite d'une étude complexe. 
En ligne avec ces constats, le présent article donne des recommandations permettant de mieux maîtriser les acquisitions IRM afin d'améliorer la qualité de la recherche multicentrique en imagerie, de la conception du protocole d'IRM, à son déploiement et à la collecte de données, en passant par le contrôle qualité des systèmes et des données, ainsi que d'en optimiser le coût et d'assurer la qualité des résultats.

\section{Remerciements :}

Nous souhaitons remercier FORCEimaging et la SFRMBM pour leur soutien moral au réseau. Nous souhaitons remercier FLI pour son soutien moral et financier.

Nous remercions tous les membres du REMI et nos mentors pour les échanges fructueux lors des réunions du réseau et en dehors.

\section{Déclaration de liens d'intérêts}

Les auteurs déclarent ne pas avoir de conflits d'intérêt.

\section{Références}

1. SFRMBM - Société Française de résonance magnétique en biologie et médecine [Internet]. [cité 26 mars 2021]. Disponible sur: https://sfrmbm.fr/

2. FLI, CERF, SFR, SFMN, CNEMBN. FORCEimaging - Réseau harmonisé de services d'imagerie pour la recherche clinique en France [Internet]. 2015 nov. Disponible sur: https://www.francelifeimaging.fr/wp-content/uploads/2016/11/FORCEi_livre_blanc.pdf

3. FLI - France Life Imaging [Internet]. FLI. [cité 26 mars 2021]. Disponible sur: https://www.francelifeimaging.fr/

4. Wiseman SJ, Meijboom R, Valdés Hernández M del C, Pernet C, Sakka E, Job D, et al. Longitudinal multi-centre brain imaging studies: guidelines and practical tips for accurate and reproducible imaging endpoints and data sharing. Trials. déc 2019;20(1):21. https://doi.org/10.1186/s13063-018-3113-6

5. Circulaire DGOS/PF4 no 2011-329 du 29 juillet 2011 relative à l'organisation de la recherche clinique et de l'innovation et au renforcement des structures de recherche clinique [Internet]. 2011. Disponible sur: https://solidaritessante.gouv.fr/fichiers/bo/2011/11-08/ste_20110008_0100_0127.pdf

6. Article L1121-1 - Code de la santé publique - Légifrance [Internet]. [cité 26 mars 2021]. 
Disponible sur:

https://www.legifrance.gouv.fr/codes/article_lc/LEGIARTI000032722870/

7. LOI $n^{\circ} 2012-300$ du 5 mars 2012 relative aux recherches impliquant la personne humaine. 2012-300 mars 5, 2012.

8. Société Française de Radiologie (SFR) [Internet]. [cité 26 mars 2021]. Disponible sur: http://www.sfrnet.org/sfr/

9. ESR | European Society of Radiology [Internet]. [cité 26 mars 2021]. Disponible sur: https://www.myesr.org/

10. Accreditation - American College of Radiology [Internet]. [cité 26 mars 2021]. Disponible sur: https://www.acr.org/Clinical-Resources/Radiology-Safety/MR-Safety

11. SpinSafety | Votre partenaire qualité et sécurité en IRM-SRM [Internet]. [cité 26 mars 2021]. Disponible sur: https://www.spinsafety.com/

12. Magphan Phantoms [Internet]. The Phantom Laboratory. [cité 26 mars 2021]. Disponible sur: https://www.phantomlab.com/magphan-phantoms

13. Keenan KE, Ainslie M, Barker AJ, Boss MA, Cecil KM, Charles C, et al. Quantitative magnetic resonance imaging phantoms: A review and the need for a system phantom: Quantitative MRI Phantoms Review. Magn Reson Med. janv 2018;79(1):48-61. https://doi.org/10.1002/mrm.26982

14. DICOM [Internet]. DICOM. [cité 26 mars 2021]. Disponible sur: https://www.dicomstandard.org

15. RĖGLEMENT (UE) 2016/ 679 DU PARLEMENT EUROPÉEN ET DU CONSEIL du 27 avril 2016 - relatif à la protection des personnes physiques à l'égard du t raitement des données à caractère personnel et à la libre circulation de ces don nées, et abrogeant la directive 95/ 46/ CE (règlement général sur la protection des données). : 88 .

16. Lam L. dicom-anonymizer: Program to anonymize dicom files with default and custom rules [Internet]. [cité 26 mars 2021]. Disponible sur: https://github.com/KitwareMedical/dicom-anonymizer

17. How to use DicomCleaner ${ }^{\mathrm{TM}}$ [Internet]. [cité 26 mars 2021]. Disponible sur: http://www.dclunie.com/pixelmed/software/webstart/DicomCleanerUsage.html

18. Esteban O, Birman D, Schaer M, Koyejo OO, Poldrack RA, Gorgolewski KJ. MRIQC: Advancing the automatic prediction of image quality in MRI from unseen sites. https://doi.org/10.1371/journal.pone.0184661

19. CNCR - Comité National de Coordination de la Recherche [Internet]. [cité 26 mars 2021]. Disponible sur: https://www.cncr.fr/

20. McNulty, Jonathan J. Fostering clinical research in imaging departments. Health Management J. 2018;18(3):222-4.

21. AFPPE_LeManipulateur_La Recherche Clinique et paramédicale. 2012;212. Disponible sur: http://new.afppe.com/downloads/2016/12/212.pdf

22. Andre JB, Bresnahan BW, Mossa-Basha M, Hoff MN, Smith CP, Anzai Y, et al. Toward Quantifying the Prevalence, Severity, and Cost Associated With Patient Motion During Clinical MR Examinations. J Am Coll Radiol. juill 2015;12(7):689-95. https://doi.org/10.1016/j.jacr.2015.03.007

23. Haute Autorité de Santé - Indicateurs pour l'amélioration de la qualité des soins Conformité des demandes d'examens d'imagerie [Internet]. 2012. Disponible sur: https://www.has-sante.fr/upload/docs/application/pdf/2013-07/ipaqss-rapportcdei_campagne2012.pdf

24. Haute Autorité de Santé - Indicateurs pour l'amélioration de la qualité des soins Conformité des demandes d'examens d'imagerie [Internet]. 2014. Disponible sur: 
https://www.has-sante.fr/upload/docs/application/pdf/2015-07/cdei_rapport_vf.pdf

25. Recueil d'activités 2013 de recherche clinique à promotion industrielle en France data.gouv.fr [Internet]. 2013 [cité 26 mars 2021]. Disponible sur: /fr/datasets/recueildactivites-2013-de-recherche-clinique-a-promotion-industrielle-en-france-1/

26. Cornu C, Binquet C, Thalamas C, Vigouroux C, Gaillard S, Ginhoux T, et al. Essais cliniques institutionnels : quel monitoring ? Therapies. mai 2013;68(3):135-41.

27. Clément O. How to foster clinical research in imaging departments - Results of the ESR Survey on European Research, 2018.

28. Mallard S. How to foster clinical research in imaging departments - How to structure a research management unit in an imaging department, 2018.

29. Y. Liu. How to foster clinical research in imaging departments - Implementing quality imaging in multicentre trials - ECR 2018.

30. McNulty J. How to foster clinical research in imaging - An overview of the roles of radiographers in research, 2018. 